\title{
Influence of the ESR saturation on the power sensitivity of cryogenic sapphire resonators
}

\author{
Vincent Giordano* and Serge Grop, Pierre-Yves Bourgeois, Yann Kersalé and Enrico Rubiola \\ FEMTO-ST Institute - UMR 6174 \\ CNRS / ENSMM / UFC / UTBM \\ 26 Chemin de l'Épitaphe \\ 25000 Besançon - FRANCE
}

(Dated: 2014 may, 12; Revised)

\begin{abstract}
Here, we study the paramagnetic ions behavior in presence of a strong microwave electromagnetic field sustained inside a cryogenic sapphire whispering gallery mode resonator. The high frequency measurement resolution that can be now achieved by comparing two CSOs permit for the first time to observe clearly the non-linearity of the resonator power sensitivity. These observations that in turn allow us to optimize the CSO operation, are well explained by the Electron Spin Resonance (ESR) saturation of the paramagnetic impurities contained in the sapphire crystal.
\end{abstract}

\section{INTRODUCTION}

Sapphire single crystal is a key material for numbers of very demanding scientific and technological applications. Even in its purest form, the sapphire single crystal always contains some paramagnetic impurities, which come from the raw material or are result of contamination during the growth process [1, 2]. Despite their low concentration these accidental dopants turn out to be very useful in some innovative systems intended for high resolution measurements. The Cryogenic Sapphire Oscillator (CSO) incorporating a cryogenic sapphire whispering gallery mode resonator is currently the most stable frequency source. It achieves in an autonomous and reliable version a relative frequency stability better than $1 \times 10^{-15}$ for integration times $\tau \leq 10,000 \mathrm{~s}$, with at long term a frequency drift of $2 \times 10^{-15} /$ day [3, 4]. The thermal compensation induced by the accidental paramagnetic impurities that substitute to $\mathrm{Al}^{3+}$ is essential for the achievement of the highest frequency stability [5, 6]. A high Q-factor microwave cryogenic resonator containing a small amount of $\mathrm{Fe}^{3+}$ can also be used as the amplifying medium to design a zero-field solid-state $12 \mathrm{GHz}$ Maser [7, 8] or as the non-linear element for microwave third harmonic generation $[9,10]$ Paramagnetic impurities in sapphire are also good candidates for the realization of quantum electrodynamics measurements[11].

In this paper we study the behavior of paramagnetic dopants diluted in a sapphire high-Q microwave resonator in which a strong electromagnetic field is sustained. The Van-Vleck model is applied to calculate the dc-magnetic susceptibility induced at low power by the different paramagnetic species that can be found in the high quality sapphire crystal. The interaction between the paramagnetic dopants and the RF electromagnetic field is described through the classical two-levels Bloch equations. We show how the saturation of the Electron
Spin Resonances (ESR) leads to a non-linear power sensitivity of the resonator frequency. Eventually the stabilisation of the injected power to the resonator turnover point allow us to optimise the oscillator frequency stability, which reaches a flicker floor of $3 \times 10^{-16}$ extending until 10,000 s.

\section{PARAMAGNETIC IONS DESCRIPTION}

\section{A. Standard magnetic susceptibility model}

The presence of paramagnetic dopants in the crystal matrice and its impact on the propagation of an electromagnetic wave at the frequency $\nu$ is accounted for through the magnetic susceptibility $\chi(\nu)=\chi^{\prime}(\nu)+$ $j \chi^{\prime \prime}(\nu) . \chi^{\prime}$ and $\chi^{\prime \prime}$ represent respectively the phase shift and the power absorption induced by the Electron Spin Resonance (ESR) [12]. Let us assume that the sapphire crystal contains a density $N$ of a paramagnetic ion presenting in its ground state two energy levels $|m\rangle$ and $|n\rangle$ separated by the ion ESR frequency $\nu_{m n}$. Solving the Bloch equations for such a two levels system interacting with the wave at the frequency $\nu$ leads for the real part of the susceptibility to a dispersive lonrentzian function that nulls at $\nu_{m n}[13]$ :

$$
\chi^{\prime}(\nu)=\chi_{0} \frac{\left(2 \pi \tau_{2}\right)^{2}\left(\nu-\nu_{m n}\right) \nu_{m n}}{1+\left(2 \pi \tau_{2}\right)^{2}\left(\nu-\nu_{m n}\right)^{2}+\Omega^{2} \tau_{1} \tau_{2}}
$$

where $\tau_{1}$ and $\tau_{2}$ are the ion spin-lattice and spin-spin relaxation times, respectively. $\Omega$ is the Rabi frequency and $\chi_{0}$ is the dc-susceptibility. Similarly, we found an absorption lorentzian lineshape for $\chi^{\prime \prime}(\nu)$, whose linewidth at low power is $\Delta \nu_{m n}=1 / \pi \tau_{2}$.

$\Omega$ is proportional to the ESR transition probability $\sigma_{m n}$ and to the RF magnetic field $B_{R F}$ :

$$
\Omega=\frac{g \mu_{B} B_{R F}}{\hbar} \sigma_{m n}
$$


where $g$ is the Landé factor, $\mu_{B}$ the Bohr Magneton and $\hbar$ the reduced Planck constant. The term $\Omega^{2} \tau_{1} \tau_{2}$ in the denominator of the equation 1 is the saturation parameter proportional to the electromagnetic power felt by the ions. This term is generally neglected in the analysis of the paramagnetic ion behavior in the CSO sapphire resonator. However, as we will see later, when the electromagnetic wave is confinded in a high-Q factor cryogenic sapphire resonator the saturation of the ESR can no longer be neglected.

The dc-susceptibility $\chi_{0}$ results from the distribution of the ions on their energy levels through the effect of the thermal agitation. The derivation of $\chi_{0}$ at a given absolute temperature $T$ is straightforward by assuming i) the energy separation between the ion ground state and excited state is large compared to $k_{B} T$, ii) the ion orbital momentum is totally quenched by the crystal field and thus the ion in the crystal lattice behaves like a free spin $S$. With these assumptions, $\chi_{0}$ follows the Curie law [14]:

$$
\chi_{0}^{C}=\mu_{0} N \frac{g^{2} \mu_{B}^{2}}{3 k_{B} T} S(S+1)
$$

where $\mu_{0}$ is the permeability of free space and $k_{B}$ the Boltzmann constant.

\section{B. On the validity of the ESR description}

The Curie law has been derived for a free system of spin $S$, which consists in $2 S+1$ levels equally spaced. Departures from the Curie law are well known for $\mathrm{Sm}^{3+}$ and $\mathrm{Eu}^{3+}$ as for these two rare-earth ions the first excited multiplet populations can not be neglected. Even for less exotic ions, in a real crystal the ground state is splitted by the crystal field in multiple degenerated Kramer's doublets separated by a Zero Field Splitting (ZFS)[15]. The table I gives the characteristics of the dominant paramagnetic species that can be found in high purity sapphire crystals.

TABLE I. ESR of the Iron-group paramagnetic ions that can be found in high-purity sapphire crystals.

\begin{tabular}{lccccccc}
\hline Ion & $S$ & $\begin{array}{c}\text { Ground state } \\
\text { transitions }\end{array}$ & $\begin{array}{c}\nu_{m n} \\
(\mathrm{GHz})\end{array}$ & $\sigma_{m n}$ & $\begin{array}{r}\tau_{1} \\
(\mathrm{~ms})\end{array}$ & $\begin{array}{r}\tau_{2} \\
(\mathrm{~ns})\end{array}$ & Ref. \\
\hline \hline $\mathrm{Cr}^{3+}$ & $3 / 2$ & $\left|\frac{1}{2}\right\rangle \rightarrow\left|\frac{3}{2}\right\rangle$ & 11.4 & 1.00 & 200 & 7 & {$[12,16]$} \\
$\mathrm{Mo}^{3+} 3 / 2$ & $\left|\frac{1}{2}\right\rangle \rightarrow\left|\frac{3}{2}\right\rangle$ & 165.0 & 2.00 & 0.1 & 12 & {$[17,18]$} \\
& & & & & & & \\
$\mathrm{Fe}^{3+}$ & $5 / 2$ & $\left|\frac{1}{2}\right\rangle \rightarrow\left|\frac{3}{2}\right\rangle$ & 12.0 & 2.00 & 10 & 20 & {$[2,19]$} \\
& & $\left|\frac{3}{2}\right\rangle \rightarrow\left|\frac{5}{2}\right\rangle$ & 19.3 & 1.25 & & & \\
& & $\left|\frac{1}{2}\right\rangle \rightarrow\left|\frac{5}{2}\right\rangle$ & 31.3 & 0.0024 & & &
\end{tabular}

Strictly speaking, for these ions the dc-susceptibility should be calculated by using the Van Vleck equation [20]:

$$
\chi_{0}^{V V}=\mu_{0} N \frac{\sum_{m}\left(E_{m}^{(1)^{2}}-2 E_{m}^{(2)}\right) \exp \left(-E_{m}^{(0)} / k_{B} T\right)}{\sum_{m} \exp \left(-E_{m}^{(0)} / k_{B} T\right)}
$$

providing we know a Taylor expansion as a function of the applied magnetic field $B_{0}$ of each populated energy levels, i.e. $E_{m}=E_{m}^{(0)}+E_{m}^{(1)} B_{0}+E_{m}^{(2)} B_{0}^{2}+\mathcal{O}^{3}$. In a number of situations, the cumbersome calculation of the Van Vleck coefficients $E_{m}^{(k)}$ is avoided and the dc-susceptibitily is assumed to follow the Curie law. However the current measurement resolution is such as it is necessary to know the degree of validity of this assumption. In [21], R. Boča uses the spin-hamiltonian formalism to derive the Van Vleck coefficients, and thus the dc-susceptibility expression for various systems presenting a ZFS. From his results we calculated $\chi_{0}^{V V}$ for $\mathrm{Cr}^{3+}, \mathrm{Fe}^{3+}$ and $\mathrm{Mo}^{3+}$ in $\mathrm{Al}_{2} \mathrm{O}_{3}$ neglecting the rhombic zero-field splitting parameter. The figure 1 shows the comparison between the Curie Law and the Van Vleck model.

In the range of temperatures reachable with a modern

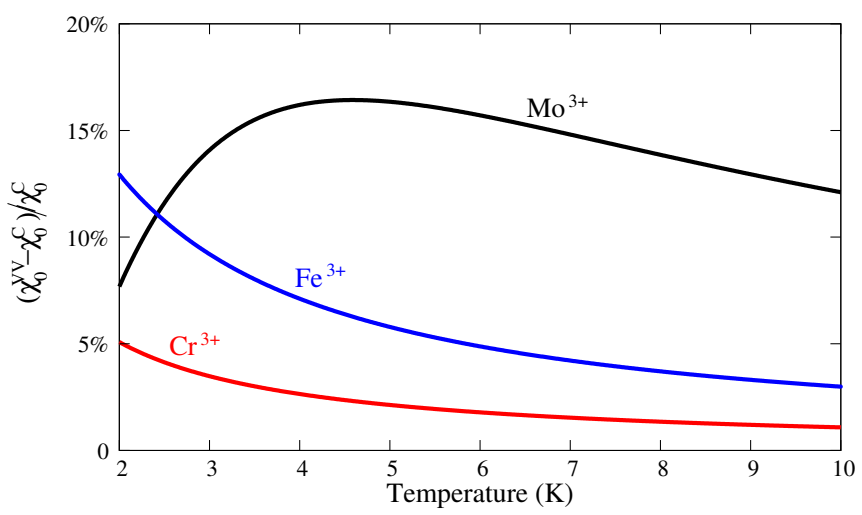

FIG. 1. Comparison between the Curie and the Van Vleck laws : $\left(\chi_{0}^{V V}-\chi_{0}^{C}\right) / \chi_{0}^{C}$ for $\mathrm{Cr}^{3+}$ (red), $\mathrm{Fe}^{3+}$ (blue) and $\mathrm{Mo}^{3+}$ (black) in $\mathrm{Al}_{2} \mathrm{O}_{3}$.

two-stages cryocooler $(3-10 \mathrm{~K})$, the difference in the two dc-susceptibility values is less than 20 percent.

A second issue in the standard model arises from the case of $\mathrm{Fe}^{3+}$ ion. The equation 1 is valid for a two levels system. $\mathrm{Fe}^{3+}$ has a spin $S=5 / 2$ and thus, its ground state is splitted in three Kramer's doublets: $|1 / 2\rangle,|3 / 2\rangle$ and $|5 / 2\rangle$. In the absence of a static magnetic field, there are thus three ESR at the frequencies $12.0 \mathrm{GHz}, 19.3 \mathrm{GHz}$ and $31.3 \mathrm{GHz}$. When dealing with a low power, the level populations stay almost those imposed by the thermal agitation. The susceptibility thermal behavior will be thus well represented by the Van Vleck model. At a high power, the differential saturation of these three transitions makes complexe the derivation of the equations. 
The $|1 / 2\rangle \rightarrow|5 / 2\rangle$ transition at $31.3 \mathrm{GHz}$ is only allowed owing the state-mixing induced by the crystal field. For a frequency $\nu$ near $10 \mathrm{GHz}$, it is thus justified to neglect this transition when calculating the evolution of overall magnetic susceptibility. About one third of the $\mathrm{Fe}^{3+}$ ion population is on the $|5 / 2\rangle$ level and is not affected by the RF magnetic field. We can reasonably conclude that the two levels model will lead to an overestimation of the impact of the ESR saturation on the magnetic susceptibility. We did not go further in the description of the $\mathrm{Fe}^{3+}$ ion behavior as the current uncertainties in the impurities concentration and in the ions relaxation times $\tau_{1}$ and $\tau_{2}$ make illusive a better quantitative analysis.

\section{WHISPERING GALLERY MODE RESONATOR: LOW POWER OPERATION.}

Due to its high-Q factor at low temperature the sapphire whispering gallery mode resonator constitutes a powerful tool to observe the behavior of the paramagnetic ions diluted in the crystal. The typical resonator geometry is shown in the figure 2 .

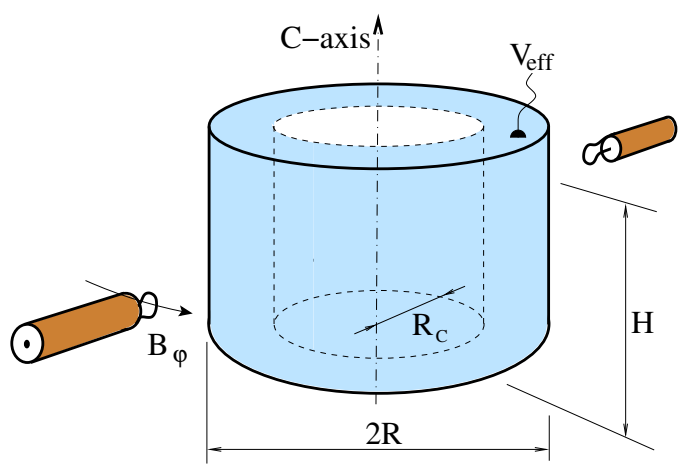

FIG. 2. The microwave whispering gallery mode resonator.

For an operation in X-band, the sapphire cylinder has a diameter $30 \leq 2 R \leq 55 \mathrm{~mm}$ and a thickness $20 \leq H \leq 30$ $\mathrm{mm}$. Its axis is parallel to the crystal C-axis within \pm 0.5 degree. The resonator is placed in the center of a cylindrical gold plated copper cavity and can be easily cooled down to about $3 \mathrm{~K}$ inside a two-stages cryocooler developping a power of $0.5 \mathrm{~W}$ at $4 \mathrm{~K}$. Two diametrically opposed small magnetic loops excite the electromagnetic resonance modes of the structure. Among them, the high order hybrid modes called whispering gallery modes are characterized by a high degree of confinement of the electromagnetic fields inside the dielectric medium. In that case the resonator quality factor is only limited by the sapphire dielectric losses which are very low at acryogenic temperature [22]. For a $10 \mathrm{GHz}$ resonance mode the typical unloaded Q-factor is one billion at $6 \mathrm{~K}$. For a whispering gallery mode, the energy density is confined between the cylindrical dielectric-air boundary and the inner caustic surface $\rho=R_{C}$ (see Fig. 2). Elsewhere the waves are evanescent. The resonator can thus be seen like a bent waveguide forming a ring [23]. The volume of this ring is $V_{e f f}=\pi H\left(R^{2}-R_{C}^{2}\right)$. In the configuration shown in the figure 2, the magnetic field generated by the loops is perpendicular to the cylindrical axis allowing to excite the quasi-transverse magnetic whispering gallery modes such as $W G H_{m, n, l}$ mode. The three integers $m, n$ and $l$ represent the electromagnetic field components variations along the azimuthal $\varphi$, radial $\rho$ and axial $z$ directions respectively [24]. We consider only the resonant modes with low radial and axial variations, i.e. those corresponding to $n=l=0$ as they present the more efficient confinement inside the dielectric medium.

At low power, the temperature dependance of a given mode frequency $\nu$ is [5]:

$$
\frac{\nu(T)-\nu_{0}}{\nu_{0}}=A T^{4}-\eta \frac{\chi^{\prime}(\nu, T)}{2}
$$

$\nu_{0}$ would be the mode frequency for a negligible circulating power, at $T=0 \mathrm{~K}$ and in the absence of any paramagnetic dopant. $A \approx-3 \times 10^{-12} \mathrm{~K}^{-4}$ [6] combines the temperature dependance of the dielectric constant and the thermal dilatation of the sapphire. The filling factor $\eta \approx 1$ for a high order whispering gallery mode. $\chi^{\prime}$ is the real part of the ac susceptibility for a RF magnetic field perpendicular to the crystal C-axis. It is the sum of the contributions of all ion species contained into the crystal. The low power assumption means that the thermal distribution of the population on the energy levels of the paramagnetic impurities is not modified by the RF magnetic field, i.e. $\Omega^{2} \tau_{1} \tau_{2} \ll 1$. For a mode frequency below the ESR of the dominant paramagnetic specie, the $1 / T$ dependance of $\chi^{\prime}$ will compensate for the intrinsic sapphire thermal sensitivity. The mode frequency passes through a maximum at a temperature $T_{0}$, which depends on the nature and concentration of the dopants. Our resonators are machined from HEMEX sapphire monocrystals provided by Crystal System Inc. [25]. Such a crystal is grown with the Heat Exchanger Method allowing the growth of large sapphire boule with the lowest defects and impurities concentration. The Fig. 3 shows the turnover temperatures as a function of the whispering gallery mode frequency for a $2 R=50 \mathrm{~mm}$ and $H=20$ mm sapphire resonator.

All whispering gallery modes in a large frequency range present a turnover temperature almost independent of the mode order $m$. Luiten [6] demonstrated that it is due to the predominance of the $\mathrm{Mo}^{3+}$ ion, whose ESR frequency is $165 \mathrm{GHz}$. The $\mathrm{Mo}^{3+}$ concentration was estimated to be of the order of some $0.1 \mathrm{ppm}$. The spread in turnover temperatures observed for low frequency modes $(\nu<12 \mathrm{GHz})$ could result from $\mathrm{Cr}^{3+}$ or $/$ and $\mathrm{Fe}^{3+}$ residuals. The concentration of these residuals should be very low as the turnover temperature imposed by the $\mathrm{Mo}^{3+}$ ions is not greatly affected. Indeed other measurements 


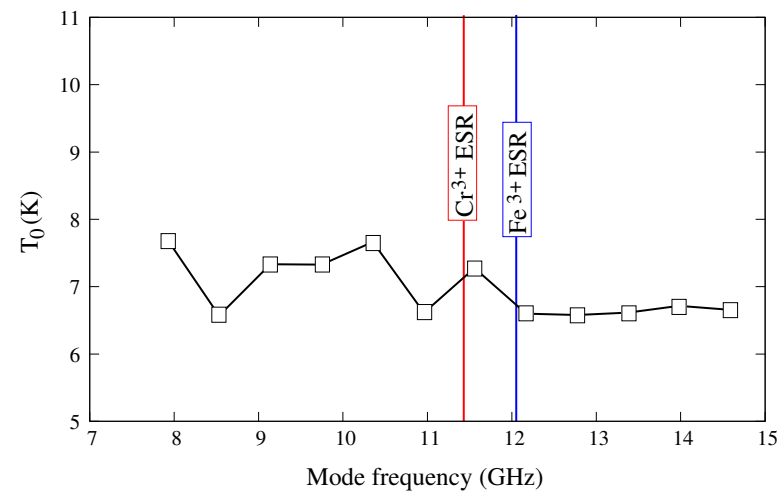

FIG. 3. $2 R=50 \mathrm{~mm}, H=20 \mathrm{~mm}$ HEMEX resonator: turnover temperature $T_{0}$ as a function of the mode frequency for $W G H_{m, 0,0}$ modes with $4 \leq m \leq 21$.

show that $\mathrm{Cr}^{3+}$ and $\mathrm{Fe}^{3+}$ concentrations are of some tens of $\mathrm{ppb}[2,26]$. The figure 4 shows the temperature dependance of the $\mathrm{WGH}_{16,0,0}$ mode at $11.565 \mathrm{GHz}$ of the same resonator and the theoretical predictions. The equation 5 evaluated with $\chi_{0}^{V V}$ and $0.15 \mathrm{ppm}$ of $\mathrm{Mo}^{3+}$ representes well the experimental frequency variation shown in the figure 4

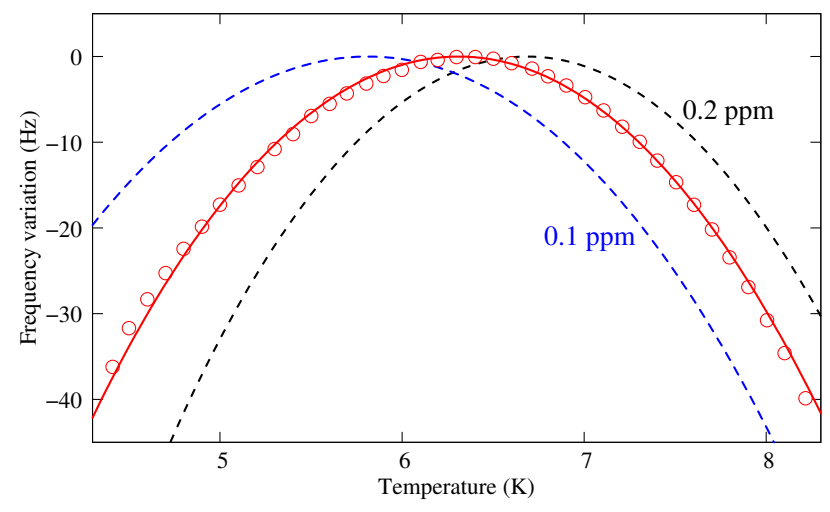

FIG. 4. $\mathrm{WGH}_{16,0,0}$ mode frequency vs temperature variation for a $50 \mathrm{~mm}$ diameter and $20 \mathrm{~mm}$ hight HEMEX sapphire resonator. Red open circles: experimental data. Dashed lines: Frequency calculated with the Van Vleck model with a $\mathrm{Mo}^{3+}$ ions concentration of $0.1 \mathrm{ppm}$ (blue dashed line), $0.2 \mathrm{ppm}$ (black dashed line) and $0.15 \mathrm{ppm}$ (solid red line).

\section{RESONATOR POWER SENSITIVITY}

In the CSO the sapphire whispering gallery mode resonator is simply inserted in the positive feedback loop of an electronic amplifier to form an oscillator as schematised in the figure 5 . The CSO is completed by two servos to stabilize the power injected into the resonator and the phase lag along the sustaining loop [27]. The error signals needed for these two servos are derived from the low frequency voltages generated by two tunnel diodes

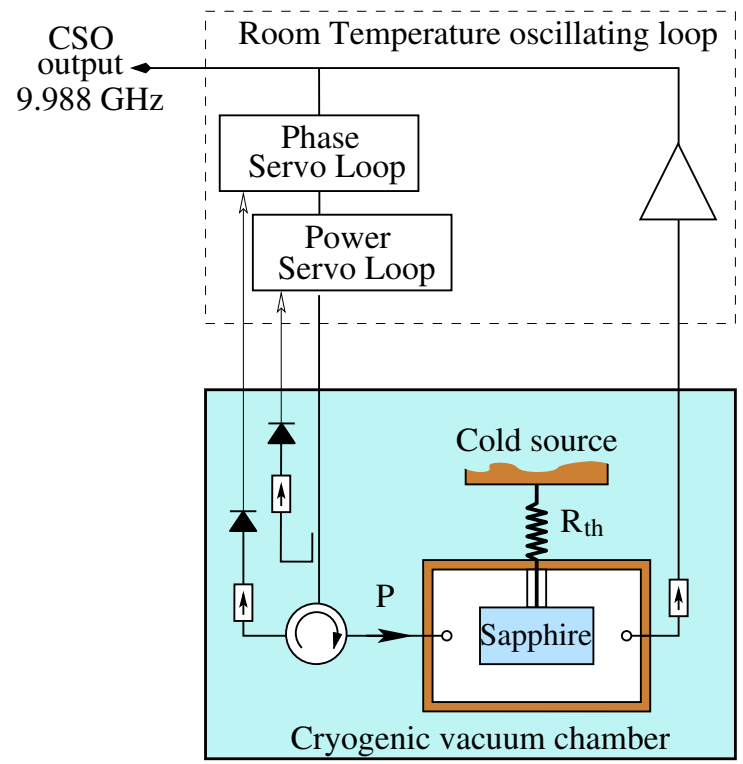

FIG. 5. Scheme of the Cryogenic Sapphire Oscillator. The cold source is a two-stages Pulse-Tube cryocooler.

placed near the resonator input port (see figure 5). We build two identical oscillators: CSO-1 and CSO-2, a third unit being under construction. These three instruments are intended to serve as references for ultra high resolution short term frequency stability measurements in the frame of the OSCILLATOR-IMP project [28]. The two CSOs are based on a $2 R=54 \mathrm{~mm}$ and $H=30$ mm HEMEX resonator designed to operate on the quasitransverse magnetic whispering gallery mode $\mathrm{WGH}_{15,0,0}$ near $10 \mathrm{GHz}$. For this resonator, the electromagnetic field is confined inside a volume $V_{\text {eff }} \approx 17 \mathrm{~cm}^{3}$. The table II gives the current resonators characteristics as measured with a network analyzer using a $-10 \mathrm{dBm}$ probe signal.

TABLE II. The two resonators parameters: $T_{0}$ : turnover temperature, $Q_{0}$ : unloaded Q-factor, $\beta_{1}$ and $\beta_{2}$ : coupling coefficient at the input and output ports respectively. $P_{0}$ : turnover power at which the resonator frequency is maximum. $\frac{\Delta \nu}{\Delta P}$ the frequency power sensitivity slope for $P \gg P_{0}$.

\begin{tabular}{|c|c|c|c|c|c|c|}
\hline & $\begin{array}{c}T_{0} \\
(\mathrm{~K}) \\
\end{array}$ & $Q_{0}$ & & $\beta_{2}$ & $\begin{array}{c}P_{0} \\
(\mu \mathrm{W})\end{array}$ & $\begin{array}{c}\frac{\Delta \nu}{\Delta P} \text { at } \\
\text { high power } \\
(\mathrm{Hz} / \mathrm{W})\end{array}$ \\
\hline CSO-1 & 6.23 & $2.0 \times 10^{9}$ & 1 & 0.1 & 120 & -91 \\
\hline CSO-2 & 6.18 & $0.7 \times 10^{9}$ & 1 & 0.1 & 300 & -39 \\
\hline
\end{tabular}

The Q-factor depends of the crystal quality but also of its cleaness. It can be affected by spurious modes and by some geometrical imperfections in the cavity symmetry or in the coupling probes alignement. Generally multiple cooldowns and fine step-by-step adjustments are required to get the highest unloaded 
Q-factor only limited by the sapphire dielectric losses. This was realized for CSO-1, still not for CSO-2 what explains its relative low Q-factor. For each resonator the coupling coefficients have been set near their optimal value, i.e. $\beta_{1} \approx 1$ and $\beta_{2} \ll 1$. The injected power $P$ is almost entirely dissipated into the resonator. When the resonator is stabilized at its turnover temperature its thermal sensitivity nulls at first order and the CSO frequency stability is no longer limited by the cold source temperature fluctuations. The current limitation in the frequency instability is not clearly established. The resonator power to frequency conversion constitutes one possible limitation [29] and needs thus to be investigated. To measure the CSO frequency sensitivity to the injected power, we follow the beatnote frequency changes when the power is varied in one CSO, while all other parameters being kept constant. Tunnel diodes placed at a low temperature turn out to be very sensitive and can be dammaged if the incident power is too high. In the current resonator implementation the maximal injected power has been limited to about $1 \mathrm{~mW}$. The figure 6 shows the relative frequency variation as a function of the injected power for CSO-1 and CSO-2. The $W G H_{15,0,0}$ mode frequency passes through a

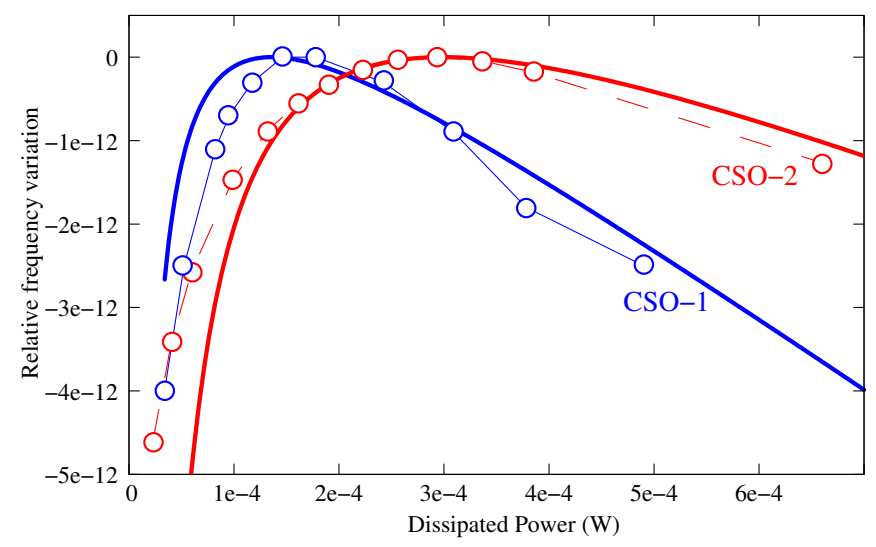

FIG. 6. Relative frequency variation vs dissipated power for COS-1 (blue) and CSO-2 (red). The open circles are the experimental points. The solid bold lines are the theoretical expectations calculated from the equation 10 assuming a concentration of $0.2 \mathrm{ppm}$ of $\mathrm{Mo}^{3+}$ and $10 \mathrm{ppb}$ of $\mathrm{Cr}^{3+}$.

maximum at a given power $P_{0}$ and exhibits for $P \gg P_{0}$ a linear negative sensitivity. The slope at high power $\frac{\Delta \nu}{\Delta P}$ and $P_{0}$, which depend on the resonator, are given in the table II.

When the injected power $P$ is increased the resonator frequency will vary through different processes:

Thermal effect: the resonator is linked by a thermal resistance $R_{t h}$ to the cold source stabilized at the temperature $T_{S}$. The resonator temperature is $T=T_{S}+R_{t h} P$. In the current design $R_{t h} \approx 2 \mathrm{KW}^{-1}$.
Thus for $P=1 \mathrm{~mW}$ the increase in the resonator temperature is only $2 \mathrm{mK}$, which around the turnover temperature induces a relative frequency shift less than $5 \times 10^{-14}$. This effect will give a negligible contribution in the experimentaly accessible range of power.

Radiation pressure: the stress induced by the stored energy results in a resonator expansion and a decrease in the dielectric constant. The resulting resonator linear frequency to power sensitivity has been previously evaluated and can be written as [29, 30]:

$$
\frac{\Delta \nu}{\Delta P}=\kappa \frac{Q_{0}}{V_{e f f}}
$$

with $\kappa \approx-7.2 \times 10^{-13} \mathrm{~Pa}^{-1}$.

In the preceeding works, the radiation pressure effect was assumed to be the major cause of the resonator power sensitivity. Indeed at a high power, this linear power dependance dominates the resonator frequency power sensibility. Equation 6 gives a sensitivity of -85 $\mathrm{HzW}^{-1}$ and $-40 \mathrm{HzW}^{-1}$ for CSO-1 and CSO-2 respectively, which are compatible with the experimental observations (see table II).

ESR saturation: the third effect arises from the saturation of the ESR. To get an approximation of the RF magnetic field seen by the ions we neglect its space variations. Over the effective volume $V_{e f f}$, we take it as a constant and equals to its mean value $\bar{B}$ defined as :

$$
\bar{B}^{2}=\frac{1}{V_{e f f}} \iiint B B^{\star} d v
$$

The stored energy is proportionnal to the power dissipated inside the resonator:

$$
E_{\text {stored }}=\mu_{0} \bar{B}^{2} V_{\text {eff }}=\frac{Q_{0} P}{2 \pi \nu_{0}}
$$

The amplitude of the ac-magnetic field can be thus written as:

$$
\bar{B}=\sqrt{\frac{\mu_{0} Q_{0} P}{2 \pi \nu_{0} V_{e f f}}}
$$

With the typical resonator parameters, i.e. $Q_{0}=10^{9}$, $P=1 \mathrm{~mW}, \nu_{0}=10 \mathrm{GHz}$ and $V_{\text {eff }}=17 \mathrm{~cm}^{3}$, we find $\bar{B}=1 \mathrm{mT}$, which is about three orders of magnitude higher than the transverse magnetic field existing inside a $50 \Omega$ coaxial cable where a $1 \mathrm{~mW}$ microwave signal is propagating. For a signal frequency $\nu$ not too far from the ESR frequency, i.e. $\left|\nu-\nu_{m n}\right| \leq$ few $\Delta \nu_{m n}$, the saturation of the ESR arises very rapidly when the injected power is increased. The ion energy level populations tend to balance themselves and the induced magnetic susceptibility goes to zero. For a signal power higher than $P_{0}$ only remains the linear power sensitivity imposed by the radiation pressure effect. 
The equation 5 is now adapted to represent the resonator sensitivity to the injected power $P$ :

$$
\frac{\nu(P)-\nu_{0}}{\nu_{0}}=A\left(T_{S}+R_{t h} P\right)^{4}+\frac{\kappa}{\nu_{0}} \frac{Q_{0}}{V_{e f f}} P-\eta \frac{\chi^{\prime}(\nu, P)}{2}
$$

This equation has been used to compute the relative frequency variation as a function of the dissipated power. The result is given in the figure 6 (bold lines) assuming a concentration of $0.2 \mathrm{ppm}$ of $\mathrm{Mo}^{3+}$ and $10 \mathrm{ppb}$ of $\mathrm{Cr}^{3+}$ for both resonators. We found about the same shape in the power dependances by replacing chromium by $1 \mathrm{ppb}$ of $\mathrm{Fe}^{3+}$. As previously mentionned, the model overestimates the saturation of the ESR for $\mathrm{Fe}^{3+}$, whose concentration has been measured of the order of $10 \mathrm{ppb}$ in similar sapphire crystals. It should also be pointed out that the value of $P_{0}$ is greatly dependant of the relaxation times $\tau_{1}$ and $\tau_{2}$, which are not known with accuracy. Nevertheless our model explains qualitatively well the power sensitivity of the sapphire resonator.

\section{APPLICATION TO THE REALIZATION OF AN ULTRA STABLE OSCILLATOR}

We can conclude from the previous observations (see figure 6) that it exists for each resonator a value of the injected power, i.e. $P_{0}$ for which the sensitivity to the power fluctuations nulls to the first order. At that point the CSO frequency stability would not any more be limited by the fluctuations of the injected power. To verify this assumption, we conducted the following measurement: CSO-2 was operated in a degraded mode with its power servo in open loop. A laboratory DC-power supply was used to bias the voltage controlled attenuator (VCA) placed in the oscillator loop and that controls the level of the injected power. The DC voltage $V$ generated by the power supply fluctuates with time following a random walk process. We observed that its standard deviation $\sigma_{V}(\tau)$ when averaged over $\tau=1 \mathrm{~s}$ is of the order of 10 $\mu \mathrm{V}$. At longer integration time, i.e. $\tau \geq 10 \mathrm{~s}, \sigma_{V}(\tau)$ is degraded proportionally to $\tau^{1 / 2}$, typical of a random walk process. Through the VCA the power injected in the resonator and thus the CSO frequency are modulated by this voltage noise. The figure 7 shows the relative frequency stability (Allan standard deviation) mesured by beating the two CSOs for different values of the power injected in CSO-2 imposed by the DC-voltage $V$. CSO-1 was nominally running with its power servo on.

When the injected power is tuned to $P_{0}=300 \mu \mathrm{W}$, the gain of the phase servo is optimal and the measured short term frequency stability at $\tau=1 \mathrm{~s}$ is $1.5 \times 10^{-15}$, which includes the contributions of both CSOs. At this level of power the CSO frequency stability would not be limited by the fluctuations of the injected power. Indeed, the relative frequency stability improves as $\tau$ is increased

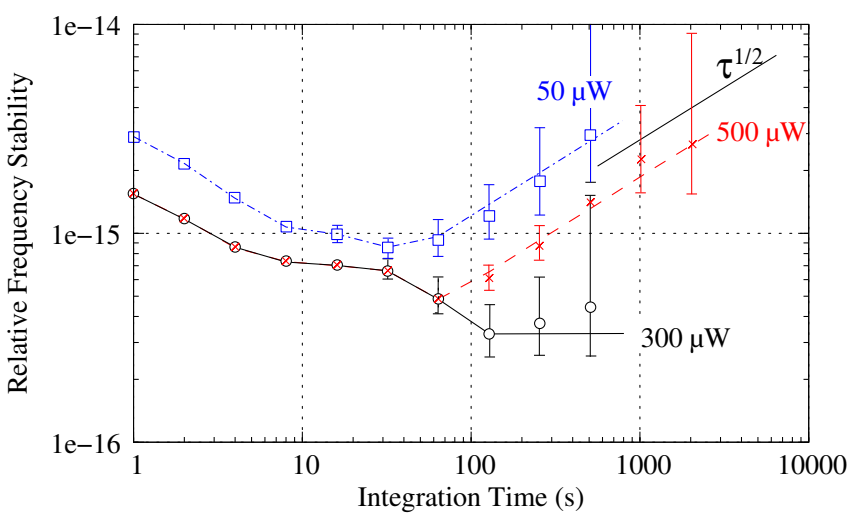

FIG. 7. Measured frequency stability (Allan standard deviation) for different values of the power injected in CSO-2. CSO-2 operated in a degraded mode without power control.

to reach a flicker floor of $3 \times 10^{-16}$. At a high power, i.e. $P=500 \mu \mathrm{W}$, the short frequency stability remains unaltered. Then for $\tau \geq 100 \mathrm{~s}$, the frequency stability is clearly limited by random walk process. The measured frequency noise level is compatible with the DCvoltage noise and the resonator sensitivity at such a high injected power. At low injected power, $P=50 \mu \mathrm{W}$, the short term frequency stability for $\tau \leq 10 \mathrm{~s}$ is degraded as the gain of the phase servo, which is proportional to $P$, is decreased. At longer integration time $\tau \geq 100$ s, the measured frequency stability is degraded proportionally to $\tau^{1 / 2}$ with a frequency noise level higher than for $P=500 \mu \mathrm{W}$ as the power sensitivity at low injected power is higher.

Eventually, in both oscillators, the resonator temperature and the injected power were stabilized at their inversion point, i.e. $T_{0}$ and $P_{0}$ respectively. The relative frequency stability is measured by beating the signals of the two identical CSOs, separated by $7.029 \mathrm{MHz}$, with a frequency counter without dead time. The next figure shows the raw data recorded for a quiet period of four hours.

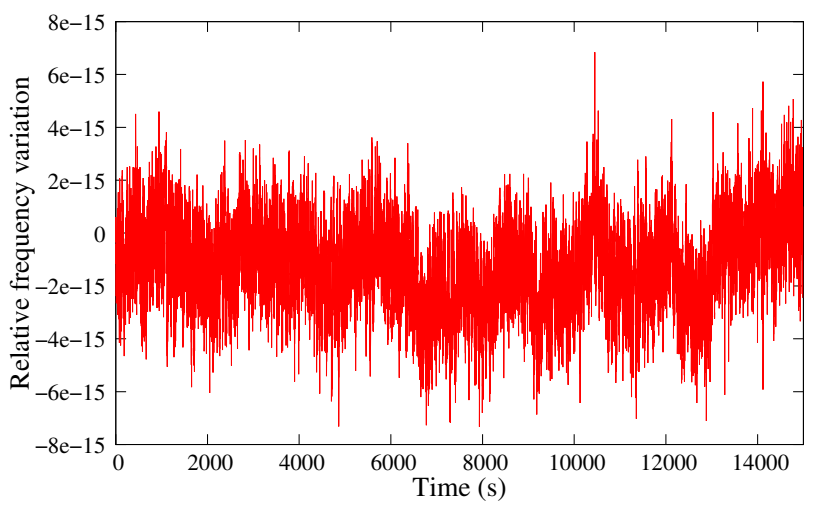

FIG. 8. Relative frequency variation during a periode of about four hours measured by beating two almost identical optimized CSOs. 
The relative frequency stability (Allan standard deviation) of one CSO is presented in the figure 9: 3 $\mathrm{dB}$ was substracted considering that the total measured noise is the sum of the contribution of two identical oscillators.

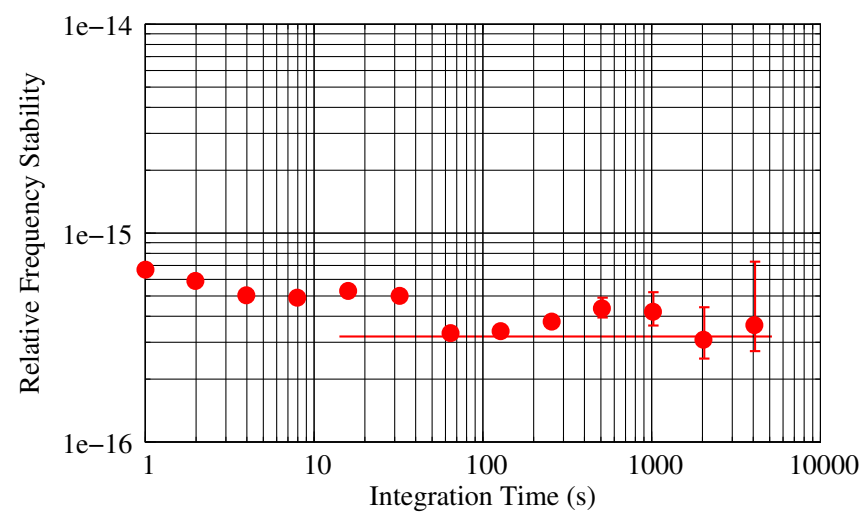

FIG. 9. Relative frequency stability of one optimized CSO. The Allan deviation has been calculated from the raw data of the figure 8 and thanks to the freeware SigmaTheta [31] available on [32] .

For $1 \mathrm{~s} \leq \tau \leq 10,000 \mathrm{~s}$ the relative frequency stability is better than $7 \times 10^{-16}$. A flicker floor of $3 \times 10^{-16}$ is reached at $100 \mathrm{~s}$. The first small hump appearing around $\tau=30 \mathrm{~s}$ could be due to a residual pumping we have detected in the temperature servo of one resonator. The second hump around $\tau=800 \mathrm{~s}$ comes from the pumping of the laboratory climatisation. This second hump could be due to the frequency stability measurement instrument sensitivity to the room temperature.

\section{CONCLUSION}

In summary we exploited the Van Vleck model to describe the saturation of the electron spin resonance of the paramagentic species contained in a high quality sapphire resonator. The proposed model explains qualitatively well the frequency-to-power sensitivity of the sapphire resonator. The ESR saturation and the sapphire intrinsic sensitivity compensate themselves at a given injected power, leading to a turnover in the frequency-vs-power curve. It is thus demonstrated than the CSO stabilized at this power value presents an exceptional short term frequency stability better than $7 \times 10^{-16}$ for $\tau \leq 10,000$ s.

\section{ACKNOWLEDGEMENTS}

The work has been realized in the frame of the ANR projects: Equipex Oscillator-Imp and Emergence ULISS-
2G. The authors would like to thank the Council of the Région de Franche-Comté for its support to the Projets d'Investissements d'Avenir and the FEDER for funding one CSO.

* giordano@femto-st.fr

[1] B. C. Barish, G. Billingsley, J. Camp, W. P. Kells, G. H. Sanders, S. E. Whitcomb, L. Y. Zhang, R.-Y. Zhu, P. Z. Deng, J. Xu, G. Q. Zhou, and Y. Z. Zhou, IEEE Transactions on Nuclear Science, 49, 1233 (2002).

[2] K. Benmessai, W. G. Farr, D. L. Creedon, Y. Reshitnyk, J.-M. L. Floch, T. Duty, and M. E. Tobar, Physical Review B, 094412 (2013).

[3] S. Grop, W. Schafer, P.-Y. Bourgeois, Y. Kersalé, M. Oxborrow, E. Rubiola, and V. Giordano, IEEE Transactions on Ultrasonics, Ferroelectrics and Frequency Control, 58 (2011).

[4] V. Giordano, S. Grop, B. Dubois, P.-Y. Bourgeois, Y. Kersalé, E. Rubiola, G. Haye, V. Dolgovskiy, N. Bucalovicy, G. D. Domenico, S. Schilt, J. Chauvin, and D. Valat, Review of Scientific Instruments, 83 (2012).

[5] A. Mann, A. Giles, D. Blair, and M. Buckingham, J. Phys. D: Appl. Phys., 25, 1105 (1992).

[6] A. N. Luiten, A. Mann, and D. Blair, J. Phys. D: Appl. Phys., 29, 2082 (1996).

[7] P. Bourgeois, N. Bazin, Y. Kersalé, V. Giordano, M. Tobar, and M. Oxborrow, Applied Physics Letters, 87 (2005), 224104(3 pages).

[8] K. Benmessai, D. Creedon, M. Tobar, P. Bourgeois, Y. Kersalé, and V. Giordano, Physical Review Letters, 100 (2008).

[9] D. L. Creedon, K. Benmessai, W. P. Bowen, and M. E. Tobar, Physical Review Letters, 108, 093902 (2012).

[10] D. L. Creedon, K. Benmessai, and M. E. Tobar, Physical Review Letters, 109, 143902 (2012).

[11] W. G. Farr, D. L. Creedon, M. Goryachev, K. Benmessai, and M. E. Tobar, Physical Review B, 88, 224426 (2013).

[12] A. E. Siegman, Microwave Solid-state Maser (McGrawHill, New York, 1964).

[13] J. Vanier and C. Audoin, The Quantum Physics of Atomic Frequency Standards, Vol I (Adam Hilger, Bristol, UK, 1989).

[14] K. H. J. Buschow and F. R. de Boer, Physics of Magnetism and Magnetic Materials (Kluwer academic publishers, New York, 2003).

[15] N. Majlis, Quantum Theory of Magnetism (World Scientific Publishing, 2007).

[16] K. J. Standley and R. A. Vaughan, Physical Review, 139, 355 (1965).

[17] E. G. Sharoyan, O. S. Torosyan, E. A. Markosyan, and V. T. Gabrielyan, Physica Status Solidi (b), 65 (1974).

[18] K. N. Kocharyan, A. A. Mirzakhanyan, and E. G. Sharoyan, Physica Status Solidi (b), 94, 129 (1979).

[19] G. S. Bogle and H. F. Symmons, Proc. Phys. Soc., 73, 531 (1959).

[20] J. H. V. Vleck, Science, 201, 113 (1978).

[21] R. Boča, Coordination Chemistry Reviews, 248, 757 (2004). 
[22] V. Branginsky, V.S.Ilchenko, and K. Bagdassarov, Phys. Letters A, 120, 300 (1987).

[23] P. Bourgeois and V. Giordano, IEEE Trans. on Microwave Theory and Techniques, 53, 3185 (2005).

[24] D. Cros and P. Guillon, IEEE Transactions on Microwave Theory and Techniques, 38, 1667 (1990).

[25] F. Schmid and D. Viechnicki, Solid State Technology, 9 (1973).

[26] R. Kovacich, A. Mann, and D. Blair, J. Phys. D: Appl. Phys., 30, 3146 (1997).

[27] S. Grop, P.-Y. Bourgeois, N. Bazin, Y. Kersalé, E. Rubiola, C. Langham, M. Oxborrow, D. Clapton, S. Walker, J. D. Vicente, and V. Giordano, Review of Scientific
Instruments, 81, 025102 (2010).

[28] http://www.oscillator-imp.com/ ().

[29] N. R. Nand, S. R. Parker, E. N. Ivanov, J.-M. Le Floch, J. G. Hartnett, and M. E. Tobar, Applied Physics Letters, 103, 043502 (2013).

[30] S. Chang, A. G. Mann, A. N. Luiten, and D. G. Blair, Physical Review Letters, 79, 2141 (1997).

[31] F. Vernotte and E. Lantz, IEEE Transactions on Ultrasonics, Ferroelectrics and Frequency Control, 59, 523 (2012).

[32] http://theta.obs-besancon.fr/spip.php?article103\&lang=fr (). 